\title{
Enamino esters in the synthesis of heterocyclic systems. Transformation of diethyl acetone-1,3-dicarboxylate into poly- substituted 1,2,7,8-tetrahydro-2,7-naphthyridine-4-carboxylates
}

\author{
Klemen Pirnat, Petr Šimunek, ${ }^{a}$ Uroš Uršič, Jure Bezenšek, Uroš Grošelj, Amalija Golobič, \\ Anton Meden, Jurij Svete, and Branko Stanovnik*
}

Faculty of Chemistry and Chemical Technology, University of Ljubljana, Aškerčeva 5, P. O. Box 537, 1000 Ljubljana, Slovenia

${ }^{a}$ On leave from Department of Organic Chemistry and Technology, Faculty of Chemical

Technology, University of Pardubice, 53210 Pardubice, Czech Republik

E-mail: Branko.Stanovnik@fkkt.uni-lj.si

\section{Dedicated to Professor Dr. Heinz Heimgartner, University of Zürich, Switzerland, on the occasion of his $70^{\text {th }}$ anniversary}

\begin{abstract}
A simple three step synthesis of aminosubstituted 1,2,7,8-tetrahydro-2,7-naphthyridine-4carboxylates is described. Ethyl (3-cyano-6-ethoxy-2-oxo-1,2-dihydropyridin-4-yl)acetate 3, formed by the condensation of diethyl acetone-1,3-dicarboxylate with malononitrile, was transformed with $N, N$-dimethylformamide dimethylacetal (DMFDMA) into (E)-ethyl 2-(3cyano-6-ethoxy-2-oxo-1,2-dihydropyridin-4-yl)-3-(dimethylamino)propenoate $4 . \quad$ In further reaction the dimethylamino group was substituted with amino, hydroxy or substituted hydrazino group, followed by cyclisation to afford substituted 1-amino-7,8-dihydro-2,7-naphthyridine-4carboxylate 6b, 1-imino-7,8-dihydro-1H-pyrano[3,4-c]pyridine-4-carboxylate 8, and 1-imino-2aminosubstituted 1,2,7,8-tetrahydro-2,7-naphthyridine-4-carboxylates 13a-i.
\end{abstract}

Keywords: Enaminoesters, ethyl (3-cyano-6-ethoxy-2-oxo-1,2-dihydropyridin-4-yl)acetate, $(E)$ ethyl 2-(3-cyano-6-ethoxy-2-oxo-1,2-dihydropyridin-4-yl)-3-(dimethylamino)propenoate, 1,2, 7,8-tetrahydro-2,7-naphthyridine-4-carboxylates

\section{Introduction}

It has been reported that by the condensation of malononitrile $\mathbf{1}$ with diethyl acetone-1,3dicarboxylate 2 ethyl (3-cyano-6-ethoxy-2-oxo-1,2-dihydropyridin-4-yl)acetate 3 is formed. This has been transformed with $N, N$-dimethylformamide dimethylacetal (DMFDMA) into the 
corresponding (E)-ethyl 2-(3-cyano-6-ethoxy-2-oxo-1,2-dihydropyridin-4-yl)-3-(dimethylamino) -propenoate $4^{1}$ (Scheme 1).

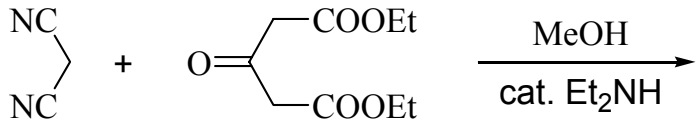

1<smiles>CCOC(=O)Cc1cc(OCC)[nH]c(=O)c1C#N</smiles>

3

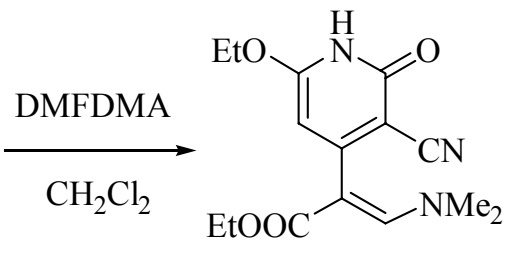

4

\section{Scheme 1}

In connection with our interest in the synthesis of various heterocyclic systems ${ }^{2,3}$ and natural products ${ }^{4}$ compound 4 , as a polyfunctional enamino ester, seems to be an excellent starting compound for the synthesis of 2,7-naphthyridine derivatives. The first preparation of 2,7naphthyridines has been been reported in $1958 .{ }^{5}$ There are several reviews dealing with the synthesis and application of 2,7-naphthyridines. ${ }^{6}$ Only few synthetic methods have a wide scope and generality. The cyclization of $o$-cyano-pyridineacetonitriles or -acetaldehyde equivalent derivatives, ${ }^{7,8}$ and a general synthesis of naphthyridines and their $N$-oxides starting from bromopyridines followed by electrophilic substitution with DMF, conventional palladiumcatalyzed cross-coupling with acetylenes, and reaction with ammonia have been published, recently. ${ }^{9,10}$ In this communication we extend the application of enamino esters to the synthesis of 1,2,7,8-tetrahydro-2,7-naphthyridine-4-carboxylates.

\section{Results and Discussion}

In the reaction of $(E)$-ethyl 2-(3-cyano-6-ethoxy-2-oxo-1,2-dihydropyridin-4-yl)-3-(dimethylamino)propenoate 4 with a mixture of aqueous ammonia and ethanol under reflux the dimethylamino group was substituted with an amino group to give intermediate 5, which, without isolation cyclized into the 1-aminonaphthyridine derivative $\mathbf{6 b}$. Similarly, heating of $\mathbf{4}$ in a mixture of aqueous solution of hydrochloric acid in methanol substitution of dimethylamino group by hydroxy group took place to form the intermediate 7, which was, without isolation, cyclized into $1 H$-pyrano[3,4-c]pyridine-4-carboxylate 8. In the reaction with aniline only substitution of the dimethylamino group occurred to give the corresponding acrylate derivative, as a 1:1 mixture of $(E)-9 \mathbf{a}$ and $(Z)-9 \mathbf{b}$ isomers, which did not cyclize by further heating. In the reaction of 4 with aryl- and heteroarylhydrazines 10a-i in methanol in the presence of catalytic amounts of hydrochloric acid substitution of the dimethylamino group took place to give intermediates 11, which were, without isolation, further cyclized under reaction conditions. Two types of products could be formed. Cyclization could occur involving either the ester group to form pyrazolidinone derivatives 12, or involving the cyano group forming naphthyridine 
derivatives 13, respectively. Since the ester group is present in the final product as shown by IR and ${ }^{1} \mathrm{H}$ NMR spectra, while the cyano group is absent in IR spectra, one can conclude that 2,7naphthyridine derivatives $\mathbf{1 3}$ were formed. In these reactions always trace amounts of 1-amino derivative $\mathbf{6 b}$ and pyrano[3,4-c]pyridine derivative $\mathbf{8}$ are formed as side products. In the reaction of 4 with benzylhydrazine in the presence of aqueous hydrochloric acid in methanol only a mixture of benzylidenehydrazinylacrylate $\mathbf{1 4}$ and $\mathbf{8}$ was formed (Scheme 2).<smiles>CCOC(=O)c1c[nH]c(=N)c2c(=O)[nH]c(OCC)cc12</smiles><smiles>[TeH]</smiles><smiles></smiles>

5<smiles>[Z17][Z10](=O)OC(=O)c1cc(OCC)[nH]c(=O)c1C#N</smiles>

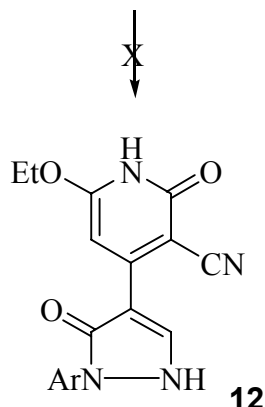<smiles>C=C=Cc1cc2c(C(=O)OCC)cnc(N)c2c(=O)[nH]1</smiles>

6b<smiles>CCOC(=O)/C(=C/Nc1ccccc1)c1cc(OCC)[nH]c(=O)c1C#N</smiles>

9a<smiles>C=CCOc1cc(/C(=C/Nc2ccccc2)C(=O)OCC)c(C#N)c(=O)[nH]1</smiles><smiles>CCOC(=N)c1c(C(=O)OCC)cc(OCC)[nH]c1=O</smiles>

$\mathrm{EtOH}, \mathrm{HCl}$ reflux, 20 min.

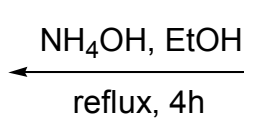<smiles>CCOC(=O)/C(=C/NC)c1cc(OCC)[nH]c(=O)c1C#N</smiles><smiles></smiles><smiles>CCOC(=O)/C=C(\C)C(=O)OCC</smiles>

7

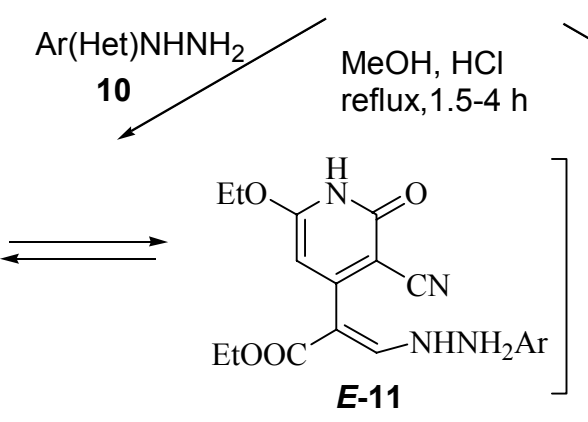<smiles>CCOC(=O)c1cn(NC(=O)c2ccccc2)c(=N)c2c(=O)[nH]c(OCC)cc12</smiles>

$10,11,12,13 \quad$ Ar

$\mathrm{Ph}$ 4- $\mathrm{NO}_{2} \mathrm{C}_{6} \mathrm{H}_{4}$ 4- $\mathrm{Cl}-\mathrm{C}_{6} \mathrm{H}_{4}$ 3-Cl- $\mathrm{C}_{6} \mathrm{H}_{4}$ 6-Cl-pyridazin-6-yl 6-Ph-pyridazin-6-yl imidazo[1,2-b]pyridazin-6-yl phthalazin-1-yl pyridin-2-yl

\section{Scheme 2}


The structures were confirmed by ${ }^{1} \mathrm{H}$ NMR and IR spectra, MS and elemental analyses for C, $\mathrm{H}$, and N. ${ }^{1} \mathrm{H}$ NMR spectra of compounds 13a-i exhibit besides typical signals for ethoxy group at position 6, ethyl ester group at position 4 and protons of aromatic or heteroaromatic groups attached at position 2, a singlet at $\delta=7.90-8.19 \mathrm{ppm}$ for $\mathrm{H}_{3}$ and a singlet at $\delta=6.69-7.14 \mathrm{ppm}$ for $\mathrm{H}_{5}$. For compound 6 the structure was confirmed also by X-ray analysis showing that this compound exists in the 1-amino form $\mathbf{6 b}$ and not in 1-imino tautomeric form $\mathbf{6 a}$ (Figure 1).

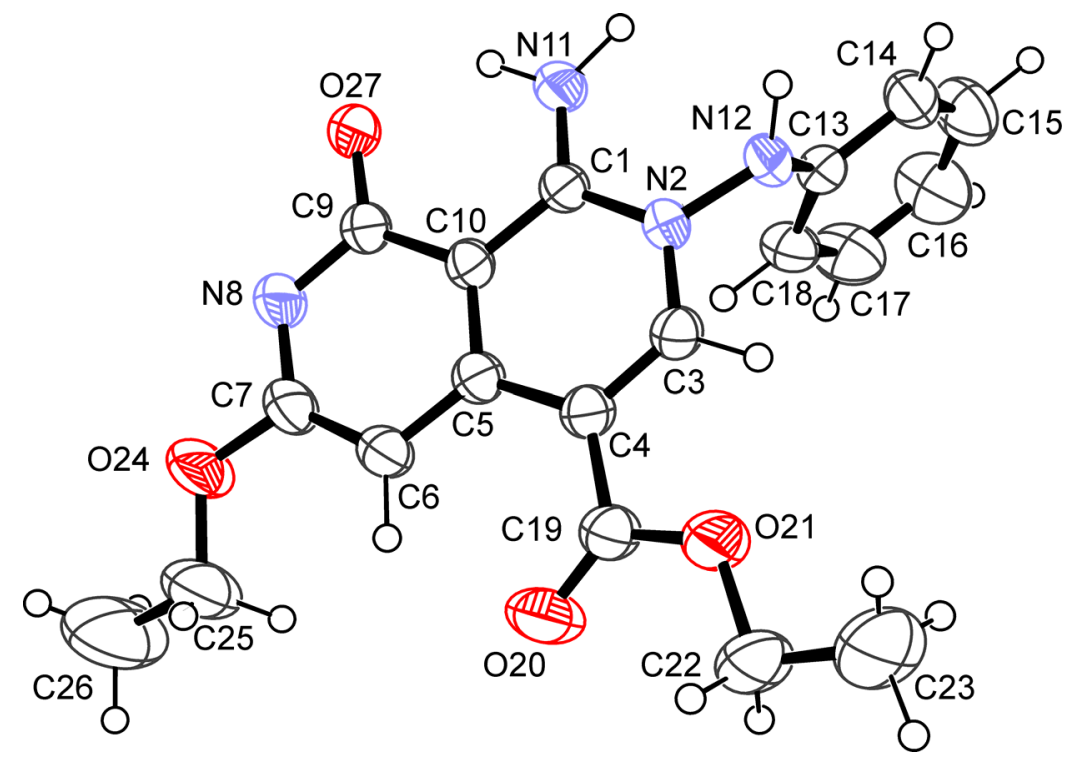

Figure 1. Ortep view of compound $\mathbf{6 b}$ at the $50 \%$ probability level of ellipsoids. $H$ atoms are drawn as small spheres of arbitrary radii.

\section{Conclusions}

A simple three step synthesis of substituted 1,2,7,8-tetrahydro-2,7-naphthyridine-4-carboxylates 6b and 13a-i by transformation of ethyl (3-cyano-6-ethoxy-2-oxo-1,2-dihydropyridin-4yl)acetate 3, formed by the condensation of diethyl acetone-1,3-dicarboxylate with malononitrile, with $N, N$-dimethylformamide dimethylacetal (DMFDMA) into (E)-ethyl 2-(3-cyano-6-ethoxy-2oxo-1,2-dihydropyridin-4-yl)-3-(dimethylamino)propenoate 4 followed by treatment with ammonia or monosubstituted hydrazines was developed.

\section{Experimental Section}

General. Melting points were taken with a Kofler micro hot stage. The ${ }^{1} \mathrm{H}$ NMR (300 MHz) and ${ }^{13} \mathrm{C}(75 \mathrm{MHz})$ spectra were obtained with a Bruker Avance DPX $300(300 \mathrm{MHz})$ spectrometer with DMSO- $\mathrm{d}_{6}$ as solvent and $\mathrm{Me}_{4} \mathrm{Si}$ as internal standard. IR spectra were recorded with a 
Perkin-Elmer Spectrum BX FTIR spectrophotometers (KBr discs). The microanalyses for C, H, and $\mathrm{N}$ were obtained with a Perkin-Elmer CHN Analyser 2400 II. Reactions were followed by TLC: Merck, Alufolien Kieselgel 60 F 254, 0.2 mm. (E)-ethyl 2-(3-cyano-6-ethoxy-2-oxo-1,2dihydropyridin-4-yl)-3-(dimethylamino)propenoate 4 was prepared according to the literature procedure. $^{1}$

Ethyl 6-ethoxy-1-amino-8-oxo-7,8-dihydro-2,7-naphthyridine-4-carboxylate

(6b). Compound 4 (153 mg, $0.5 \mathrm{mmol})$ and ammonia (25\% aqueous solution, $2 \mathrm{~mL})$ in EtOH (4 mL) was heated under reflux for $4 \mathrm{~h}$. The precipitate was after cooling collected by filtration, washed with hot EtOH. Yield $140 \mathrm{mg}(72 \%)$, yellow crystals, m.p. $300-303{ }^{\circ} \mathrm{C}\left(\mathrm{CHCl}_{3} / \mathrm{DMF}\right) .{ }^{1} \mathrm{H} \mathrm{NMR}$ $\left(\mathrm{DMSO}-d_{6}\right): \delta 1.31\left(\mathrm{t}, 3 \mathrm{H}, J=6.9 \mathrm{~Hz}, \mathrm{OCH}_{2} \mathrm{CH}_{3}\right) ; 1.37\left(\mathrm{t}, 3 \mathrm{H}, J=6.9 \mathrm{~Hz}, \mathrm{OCH}_{2} \mathrm{CH}_{3}\right) ; 4.22(\mathrm{~m}$, $\left.4 \mathrm{H}, J=6.9 \mathrm{~Hz}, 2 \times \mathrm{OCH}_{2} \mathrm{CH}_{3}\right) ; 7.02(\mathrm{~s}, 1 \mathrm{H},=\mathrm{CH}) ; 7.80($ br s, $1 \mathrm{H}, \mathrm{NH}) ; 8.62(\mathrm{~s}, 1 \mathrm{H},=\mathrm{CH}) ; 9.13$ (br s, 1H, NH); 12.10 (s, 1H, NH). IR (KBr), $\mathrm{cm}^{-1}: 3338,3157,2983,2673,1687,1619,1571$, 1509, 1464, 1368, 1304, 1269, 1183, 1131, 1074, 1027, 930, 895, 822, 725, 700, 669, 553, 457. ESI-HRMS: Calcd. for $\mathrm{C}_{13} \mathrm{H}_{15} \mathrm{~N}_{3} \mathrm{O}_{4}: \mathrm{m} / z=278,1141\left(\mathrm{MH}^{+}\right)$. Found: $\mathrm{m} / \mathrm{z}=278,1145$. Anal. Calcd. for $\mathrm{C}_{13} \mathrm{H}_{15} \mathrm{~N}_{3} \mathrm{O}_{4}$ : C, 56.31; H, 5.45; N 15.15. Found: C, 56.21; H, 5.31; N, 15.13.

Ethyl 6-ethoxy-1-imino-8-oxo-7,8-dihydro-1 $H$-pyrano[3,4-c]pyridine-4-carboxylate (8). Compound 4 (153 mg, $0.5 \mathrm{mmol})$ and hydrochloric acid (37\% aqueous solution, $0.5 \mathrm{~mL})$ in $\mathrm{MeOH}(1.5 \mathrm{~mL})$ was left at room temperature for 4 days. The precipitate was, after cooling, collected by filtration, washed with $\mathrm{MeOH}$ and recrystallized. Yield $47 \mathrm{mg}$ (34\%), m.p. 252-255 ${ }^{\circ} \mathrm{C}(\mathrm{AcOH}) .{ }^{1} \mathrm{H} \mathrm{NMR}\left(\mathrm{DMSO}-d_{6}\right): \delta 1.32\left(\mathrm{~m}, 6 \mathrm{H}, J=7.2 \mathrm{~Hz}, 2 \times \mathrm{OCH}_{2} C_{3}\right) ; 4.24(\mathrm{~m}, 4 \mathrm{H}, J=$ $\left.6.9 \mathrm{~Hz}, 2 \times \mathrm{OCH}_{2} \mathrm{CH}_{3}\right) ; 7.31(\mathrm{~s}, 1 \mathrm{H},=\mathrm{CH}) ; 8.11(\mathrm{~s}, 1 \mathrm{H},=\mathrm{CH}) ; 12.39$ (br s, $\left.1 \mathrm{H}, \mathrm{NH}\right) ; 13.63$ (s, $1 \mathrm{H}, \mathrm{NH})$. IR $(\mathrm{KBr}) \mathrm{cm}^{-1}: 3550,3477,3414,3235,2995,2932,1726,1616,1526,1439,1359$, 1308, 1269, 1233, 1112, 1036, 934, 848, 760, 617, 474. EI-HRMS: Calcd. for $\mathrm{C}_{13} \mathrm{H}_{14} \mathrm{~N}_{2} \mathrm{O}_{5}: \mathrm{m} / \mathrm{z}$ $=278,0903\left(\mathrm{M}^{+}\right)$. Found: $m / z=278,0905$. Anal Calcd for $\mathrm{C}_{13} \mathrm{H}_{14} \mathrm{~N}_{2} \mathrm{O}_{5}: \mathrm{C}, 56,11 ; \mathrm{H}, 5,07 ; \mathrm{N}$, 10,07. Found: C, 56.06; H, 5.10; N, 10.08 .

Ethyl 2-(3-cyano-6-ethoxy-2-oxo-1,2-dihydropyridin-4-yl)-3-(2-phenylamino)acrylate (9). To a solution of compound 4 (153 mg, $0.5 \mathrm{mmol})$ and aniline $(47 \mathrm{mg}, 0.5 \mathrm{mmol})$ in EtOH (2 mL) hydrochloric acid ( $37 \%$ aqueous solution, $0.2 \mathrm{~mL}$ ) was added and the mixture was heated under reflux for $20 \mathrm{~min}$. The precipitate was, after cooling, collected by filtration and recrystallized. Yield $97 \mathrm{mg}$ (55\%), yellow solid, m.p. 191-193 ${ }^{\circ} \mathrm{C}(\mathrm{EtOH}) .{ }^{1} \mathrm{H}$ NMR (DMSO- $\left.d_{6}\right): \delta 1.21(2 \times \mathrm{t}$, $\left.6 \mathrm{H}, J=7.2 \mathrm{~Hz}, \mathrm{OCH}_{2} \mathrm{CH}_{3}\right) ; 1.33\left(2 \mathrm{x} \mathrm{t}, 6 \mathrm{H}, J=7.2 \mathrm{~Hz}, \mathrm{OCH}_{2} \mathrm{CH}_{3}\right) ; 4.08-4.35$ (two overlapped q, 4H, OCH $\left.\mathrm{CH}_{3}\right) ; 6.12$ (br s, $\left.1 \mathrm{H}, \mathrm{NH}\right) ; 6.28$ (br s, $\left.1 \mathrm{H}, \mathrm{NH}\right) ; 7.06(\mathrm{~m}, 2 \mathrm{H}, \mathrm{Ph}) ; 7.17$ (d, 2H, J= $7.8 \mathrm{~Hz}, \mathrm{Ph}) ; 7.34$ (m, 6H, Ph); 7.89 (d, 1H, J=13.2 Hz, =CH); 8.04 (d, 1H, J=13.5 Hz, =CH); 8.95 (d, 1H, $J=13.8 \mathrm{~Hz}, \mathrm{NH}) ; 10.40$ (d, 1H, $J=13.2 \mathrm{~Hz}, \mathrm{NH}) ; 12.34$ (br s, 2H, NH). IR (KBr), $\mathrm{cm}^{-1}:$ 3548, 3478, 3416, 3234, 2202, 2170, 1680, 1649, 1617, 1595, 1577, 1474, 1374, 1354, 1229, 804, 760, 622, 482, 466, 412. EI-HRMS: Calcd. for $\mathrm{C}_{19} \mathrm{H}_{19} \mathrm{~N}_{3} \mathrm{O}_{4}: m / z=353,1376\left(\mathrm{M}^{+}\right)$. Found: $m / z=353,1383$. Anal Calcd. for: $\mathrm{C}_{19} \mathrm{H}_{19} \mathrm{~N}_{3} \mathrm{O}_{4}$. Anal Calc. for $\mathrm{C}_{19} \mathrm{H}_{19} \mathrm{~N}_{3} \mathrm{O}_{4}$ : C, 64.58; $\mathrm{H}$, 5.42; N, 11.89. Found: C, 64.55; H, 5.46; N, 11.77 . 


\section{Ethyl 3-(2-benzylidenehydrazinyl)-2-(3-cyano-6-ethoxy-2-oxo-1,2-dihydropyridin-4yl)acry-} late (14). A solution of compound $4(153 \mathrm{mg}, 0.5 \mathrm{mmol})$ and benzylhydrazine dihydrochloride $(132 \mathrm{mg}, 0.5 \mathrm{mmol})$ in water $(0.5 \mathrm{~mL})$ and $\mathrm{MeOH}(3 \mathrm{~mL})$ was heated under reflux for $3.5 \mathrm{~h}$. The precipitate was after cooling collected by filtration. The crude product, which is a mixture of 14 and $\mathbf{8}$ was heated with a boiling mixture of $\mathrm{AcOH} /$ toluene $(4: 1)$ in which $\mathbf{8}$ is solubile. The solid 14 was separated by filtration and recrystallized. Yellow solid, m.p. 185-188 ${ }^{\circ} \mathrm{C}$ (MeCOOH/toluene): Yield: 17\% (32 mg). ${ }^{1} \mathrm{H}$ NMR (DMSO- $\left.d_{6}\right): \delta 1.28(\mathrm{t}, 3 \mathrm{H}, J=6.9 \mathrm{~Hz}$, $\mathrm{OCH}_{2} \mathrm{CH}_{3}$ ); 1.34 (t, 3H, $\left.J=7.2 \mathrm{~Hz}, \mathrm{OCH}_{2} \mathrm{CH}_{3}\right) ; 4.26$ (q, $2 \mathrm{H}, J=6.9 \mathrm{~Hz}, \mathrm{OCH}_{2} \mathrm{CH}_{3}$ ); 4.32 (q, $\left.2 \mathrm{H}, J=6.9 \mathrm{~Hz}, \mathrm{OCH}_{2} \mathrm{CH}_{3}\right) ; 6.66(\mathrm{~s}, 1 \mathrm{H},=\mathrm{CH}) ; 7.60(\mathrm{~m}, 3 \mathrm{H}, \mathrm{Ph}) ; 8.11\left(\mathrm{dd}, 2 \mathrm{H}, J_{1}=8.1, J_{2}=1.8\right.$ $\mathrm{Hz}, \mathrm{Ph}) ; 8.65(\mathrm{~s}+$ br s, $2 \mathrm{H},=\mathrm{CH}+\mathrm{NH}) ; 9.00(\mathrm{~s}, 1 \mathrm{H},=\mathrm{CH}) ; 12.53(\mathrm{~d}, 1 \mathrm{H}, J=3.9 \mathrm{~Hz}, \mathrm{NH}) .{ }^{13} \mathrm{C}$ NMR (DMSO- $\left.d_{6}\right): \delta 14.1 ; 14.6 ; 60.5 ; 60.8 ; 88.5 ; 96.0 ; 108.9 ; 128.8 ; 129.6 ; 132.1 ; 132.5 ; 141.7$; $153.5 ; 159.3 ; 164.1 ; 168.0 ; 168.0 ; 172.1$. IR (KBr), $\mathrm{cm}^{-1}: 3476,3416,3137,2988,2903,2207$, 1678, 1648, 1588, 1474, 1378, 1345, 1311, 1236, 1055, 1023, 980, 808, 784, 761, 698, 652, 513, 463. ESI-HRMS: Calcd. for $\mathrm{C}_{20} \mathrm{H}_{20} \mathrm{~N}_{4} \mathrm{O}_{4}: m / z=381,1563\left(\mathrm{MH}^{+}\right)$. Found: $m / z=381,1567$. Anal Calcd. for $\mathrm{C}_{20} \mathrm{H}_{20} \mathrm{~N}_{4} \mathrm{O}_{4}$ : C, 63.15; H, 5.30; N, 14.73. Found: C, 63.15; H, 5.30; N, 14.73.

\section{General procedure for the synthesis of compounds (13a-i)}

A mixture of compound $4(157 \mathrm{mg}, 0.51 \mathrm{mmol})$ and aryl- or heteroarylhydrazine 10a-i $(0.54$ mmol) dissolved in $\mathrm{MeOH}(1.5$ to $4 \mathrm{~mL})$ and hydrochloric acid $(37 \%, 0.3 \mathrm{~mL})$ was heated under reflux for 1.5 to $4 \mathrm{~h}$. The yellow precipitate was, after cooling, collected by filtration and washed with $\mathrm{MeOH}$ to give analytically pure 13a-i.

The following compounds were prepared accordingly:

Ethyl 6-ethoxy-1-imino-8-oxo-2-(phenylamino)-1,2,7,8-tetrahydro-2,7-naphthyridine-4-carboxylate (13a). This compound was prepared from 4 and phenylhydrazine hydrochloride 10 in $\mathrm{MeOH}$ (1.3 mL), reflux 2.5 h. Yield: $96 \mathrm{mg}$ (51\%), yellow microcrystals, m.p. $241-243{ }^{\circ} \mathrm{C} .{ }^{1} \mathrm{H}$ NMR (DMSO-d $): \delta 1.26\left(\mathrm{~m}, 6 \mathrm{H}, 2 \times \mathrm{OCH}_{2} \mathrm{CH}_{3}\right), 4.24\left(\mathrm{~m}, 4 \mathrm{H}, 2 \times \mathrm{OCH}_{2} \mathrm{CH}_{3}\right), 6.69(\mathrm{~s}, 1 \mathrm{H}$, $=\mathrm{CH}), 6.71(\mathrm{~d}, 2 \mathrm{H}, J=7.5 \mathrm{~Hz}, \mathrm{Ph}), 6.99$ (t, 1H, $J=7.2 \mathrm{~Hz}, \mathrm{Ph}), 7.31$ (t, 2H, $J=7.8 \mathrm{~Hz}, \mathrm{Ph}), 7.90$ $(\mathrm{s}, 1 \mathrm{H},=\mathrm{CH}), 8.77(\mathrm{~d}, 1 \mathrm{H}, J=3.3 \mathrm{~Hz}, \mathrm{NH}), 9.35(\mathrm{~s}, 1 \mathrm{H}, \mathrm{NH}), 12.41(\mathrm{~d}, 1 \mathrm{H}, J=3.3 \mathrm{~Hz}, \mathrm{NH})$. IR $\left(\mathrm{KBr}, \mathrm{cm}^{-1}\right): 3434,3142,3105,3064,2982,2903,1723,1651,1606,1586,1541,1527,1445$, 1384, 1275, 1194, 1136, 1107, 1026, 925, 829, 749. EI-HRMS: Calcd. for $\mathrm{C}_{19} \mathrm{H}_{20} \mathrm{~N}_{4} \mathrm{O}_{4}: m / z=$ 368.1485 $\left(\mathrm{M}^{+}\right)$. Found: $m / z=368.1493$. Anal. Calcd. for $\mathrm{C}_{19} \mathrm{H}_{20} \mathrm{~N}_{4} \mathrm{O}_{4}: \mathrm{C}, 61.95 ; \mathrm{H}, 5.47 ; \mathrm{N}$, 15.21. Found: C, 62.12; H, 5.39; N, 15.31 .

Ethyl 6-ethoxy-1-imino-8-oxo-2-(4-nitrophenylamino)-1,2,7,8-tetrahydro-2,7-naphthyridine-4-carboxylate (13b). This compound was prepared from 4 and 4-nitrophenylhydrazine 10b in $\mathrm{MeOH}(4 \mathrm{~mL})$, reflux 4h. Yield: $130 \mathrm{mg}$ (63\%), yellow solid, m.p. 265-267 ${ }^{\circ} \mathrm{C} .{ }^{1} \mathrm{H}$ NMR $\left(\mathrm{DMSO}-d_{6}\right): \delta 12.6\left(\mathrm{~m}, 6 \mathrm{H}, J=7.8 \mathrm{~Hz}, 2 \times \mathrm{OCH}_{2} \mathrm{CH}_{3}\right), 4.25(\mathrm{~m}, 4 \mathrm{H}, J=7.2 \mathrm{~Hz}, 2 \times$ $\left.\mathrm{OCH}_{2} \mathrm{CH}_{3}\right), 6.70(\mathrm{~s}, 1 \mathrm{H},=\mathrm{CH}), 6.85(\mathrm{~d}, 2 \mathrm{H}, J=9.0 \mathrm{~Hz}, \mathrm{Ph}), 8.03(\mathrm{~s}, 1 \mathrm{H},=\mathrm{CH}), 8.18(\mathrm{~d}, 2 \mathrm{H}, J=$ $9.0 \mathrm{~Hz}, \mathrm{Ph}$ ), 8.87 (br s, 1H, NH), 10.37 (br s, 1H, NH), 12.50 (s, 1H, NH). IR (KBr cm $\left.{ }^{-1}\right): 3418$, $3077,1715,1666,1593,1528,1440,1336,1296,1269,1191,1109,930,851,812,756,644$, 
472. ESI-HRMS: Calcd. for $\mathrm{C}_{19} \mathrm{H}_{19} \mathrm{~N}_{5} \mathrm{O}_{6}: m / z=414.1414\left(\mathrm{MH}^{+}\right)$. Found: $m / z=414.1425$. Anal. Calcd. for $\mathrm{C}_{19} \mathrm{H}_{19} \mathrm{~N}_{5} \mathrm{O}_{6}$ : C, 55.20; H, 4.63; N, 16.94. Found: C, 55.10; H, 4.48; N, 16.86 .

Ethyl 6-ethoxy-1-imino-8-oxo-2-(4-chlorophenylamino)-1,2,7,8-tetrahydro-2,7-naphthyridine-4-carboxylate (13c). This compound was prepared from 4 and 4-chlorophenylhydrazine hydrochloride (10c) in $\mathrm{MeOH}(1.5 \mathrm{~mL})$, reflux $1.5 \mathrm{~h}$. Yield: $107 \mathrm{mg}$ (53\%), yellow microcrystals, m.p. $241-243{ }^{\circ} \mathrm{C}$. ${ }^{1} \mathrm{H}$ NMR (DMSO-d $): \delta 1.27(\mathrm{~m}, 6 \mathrm{H}, J=7.2 \mathrm{~Hz}, 2 \times$ $\left.\mathrm{OCH}_{2} \mathrm{CH}_{3}\right), 4.25\left(\mathrm{~m}, 4 \mathrm{H}, J=6.9 \mathrm{~Hz}, 2 \times \mathrm{OCH}_{2} \mathrm{CH}_{3}\right), 6.69(\mathrm{~s}, 1 \mathrm{H},=\mathrm{CH}), 6.74\left(\mathrm{dt}, 2 \mathrm{H}, J_{1}=8,7\right.$ $\left.\mathrm{Hz}, J_{2}=1,8 \mathrm{~Hz}, \mathrm{Ph}\right), 7.35\left(\mathrm{dt}, 2 \mathrm{H}, J_{1}=8.7 \mathrm{~Hz}, J_{2}=1.8 \mathrm{~Hz}, \mathrm{Ph}\right), 7.92(\mathrm{~s}, 1 \mathrm{H},=\mathrm{CH}), 8.78$ (br s, 1H, NH), 9.50 (s, 1H, NH), 12.43 (br s, 1H, NH). IR (KBr, cm $\left.{ }^{-1}\right): 3430,3249,3104,2977,1725$, 1707, 1647, 1546, 1492, 1439, 1380, 1297, 1275, 1243, 1177, 1095, 1032, 925, 831, 816, 648, 509. EI-HRMS: Calcd. for $\mathrm{C}_{19} \mathrm{H}_{19} \mathrm{ClN}_{4} \mathrm{O}_{4}: m / z=402.1095$. Found: $m / z=402.1103\left({ }^{35} \mathrm{Cl}, \mathrm{M}^{+}\right)$. Anal. Calcd. for $\mathrm{C}_{19} \mathrm{H}_{19} \mathrm{ClN}_{4} \mathrm{O}_{4}$ : C, 56.65; H, 4.75; N, 13.91. Found: C, 56.45; H, 4.74; N, 14.01 . Ethyl 6- ethoxy-1-imino-8-oxo-2-(3-chlorophenylamino)-1,2,7,8-tetrahydro-2,7-naphthyridine-4-carboxylate (13d). This compound was prepared from 4 and 3-chlorophenylhydrazine hydrochloride 10d in MeOH (3.0 mL), reflux 1.5 h. Yield: $88 \mathrm{mg}(44 \%)$, yellow microcrystals, m.p. $242-245^{\circ} \mathrm{C} .{ }^{1} \mathrm{H}$ NMR (DMSO-d $): \delta 1.26\left(\mathrm{~m}, 6 \mathrm{H}, J=6.9 \mathrm{~Hz}, 2 \times \mathrm{OCH}_{2} \mathrm{CH}_{3}\right), 4.24(\mathrm{~m}, 4 \mathrm{H}$, $\left.J=6.9 \mathrm{~Hz}, 2 \times \mathrm{OCH}_{2} \mathrm{CH}_{3}\right), 6.63\left(\mathrm{dt}, 1 \mathrm{H}, J_{1}=7.5 \mathrm{~Hz}, J_{2}=2.1 \mathrm{~Hz}, \mathrm{Ph}\right), 6.69(\mathrm{~s}, 1 \mathrm{H},=\mathrm{CH}), 6.78(\mathrm{t}$, $1 \mathrm{H}, J=2.1 \mathrm{~Hz}, \mathrm{Ph}), 7.02\left(\mathrm{dt}, 1 \mathrm{H}, J_{1}=7.5 \mathrm{~Hz}, J_{2}=1.8 \mathrm{~Hz}, \mathrm{Ph}\right), 7.31(\mathrm{t}, 1 \mathrm{H}, J=8.1 \mathrm{~Hz}, \mathrm{Ph}), 7.95$ $(\mathrm{s}, 1 \mathrm{H},=\mathrm{CH}), 8.78$ (br s, $1 \mathrm{H}, \mathrm{NH}) ; 9.59(\mathrm{~s}, 1 \mathrm{H}, \mathrm{NH}), 12.44(\mathrm{~s}, 1 \mathrm{H}, \mathrm{NH}) . \mathrm{IR}\left(\mathrm{KBr}, \mathrm{cm}^{-1}\right): 3420$, 2981, 1725, 1703, 1650, 1598, 1549, 1439, 1381, 1299, 1272, 1178, 1101, 1033, 929, 830, 768, 682. EI-HRMS: Calcd. for $\mathrm{C}_{19} \mathrm{H}_{19} \mathrm{ClN}_{4} \mathrm{O}_{4}: m / z=402.1095$. Found: $m / z=402.1103\left({ }^{35} \mathrm{Cl}_{1} \mathrm{M}^{+}\right)$. Anal. Calcd. for $\mathrm{C}_{19} \mathrm{H}_{19} \mathrm{ClN}_{4} \mathrm{O}_{4}$ : C, 56.65; H, 4.75; N, 13.91. Found: C, 56.53; H, 4.80; N, 13.93.

Ethyl 6-ethoxy-2-(6-chloropyridazin-3-ylamino)-1-imino-8-oxo-1,2,7,8-tetrahydro-2,7naphthyridine-4-carboxylate (13e). This compound was prepared from 4 and 3-chloro-6hydrazinopyridazine 10e in $\mathrm{MeOH}(3.0 \mathrm{~mL})$, reflux $2 \mathrm{~h}$. Yield: $99 \mathrm{mg}$ (49\%), yellow microcrystals, m.p. $>350{ }^{\circ} \mathrm{C} .{ }^{1} \mathrm{H}$ NMR $\left(\mathrm{DMSO}-\mathrm{d}_{6}\right): \delta 1.28\left(\mathrm{~m}, 6 \mathrm{H}, \mathrm{J}=7.2 \mathrm{~Hz}, 2 \times \mathrm{OCH}_{2} \mathrm{CH}_{3}\right)$, $4.25\left(\mathrm{~m}, 4 \mathrm{H}, 2 \times \mathrm{OCH}_{2} \mathrm{CH}_{3}\right), 6.73(\mathrm{~s}, 1 \mathrm{H},=\mathrm{CH}), 7.29(\mathrm{~d}, 1 \mathrm{H}, \mathrm{J}=9.3 \mathrm{~Hz}, \mathrm{Ar}), 7.76(\mathrm{~d}, 1 \mathrm{H}, \mathrm{J}=9.3$ $\mathrm{Hz}, \mathrm{Ar}), 8.19$ (s, 1H, =CH), 8.89 (br s, 1H, NH), 10.74 (br s, 1H, NH), 12.34 (br s, 1H, NH). IR $\left(\mathrm{KBr}, \mathrm{cm}^{-1}\right): 3407,3231,3079,2985,1713,1668,1655,1598,1553,1425,1385,1283,1210$, 1177, 1140, 1097, 1034, 928, 834, 818, 770, 666. EI-HRMS: Calcd. for $\mathrm{C}_{17} \mathrm{H}_{17} \mathrm{ClN}_{6} \mathrm{O}_{4}: m / z=$ 405,1078 $\left({ }^{35} \mathrm{Cl}, \mathrm{MH}^{+}\right)$. Found: $m / z=405,1083$. Anal. Calcd. for $\mathrm{C}_{17} \mathrm{H}_{17} \mathrm{ClN}_{6} \mathrm{O}_{4}: \mathrm{C}, 50.44 ; \mathrm{H}, 4.23$; N, 20.76. Found: C, 50.48; H, 4.06; N, 20.47.

Ethyl 6- ethoxy-2-(6-phenylpyridazin-3ylamino)-1-imino-8-oxo-1,2,7,8-tetrahydro-2,7-naphthyridine-4-carboxylate (13f). This compound was prepared from 4 and 3-hydrazino-6phenylpyridazine 10f in $\mathrm{MeOH}(1.5 \mathrm{~mL})$, reflux $2.5 \mathrm{~h}$. Yield: $128 \mathrm{mg}$ (57\%), yellow microcrystals, m.p. $>279-283{ }^{\circ} \mathrm{C}$ (decomp). ${ }^{1} \mathrm{H}$ NMR (DMSO-d $): \delta 1.32$ (t, 3H, $J=7.2 \mathrm{~Hz}$, $\left.\mathrm{OCH}_{2} \mathrm{CH}_{3}\right), 1.43\left(\mathrm{t}, 3 \mathrm{H}, J=6.9 \mathrm{~Hz}, \mathrm{OCH}_{2} \mathrm{CH}_{3}\right), 4.35\left(\mathrm{~m}, 4 \mathrm{H}, 2 \times \mathrm{OCH}_{2} \mathrm{CH}_{3}\right), 7.14(\mathrm{~s}, 1 \mathrm{H},=\mathrm{CH})$, 7.54 ( $\mathrm{m}, 3 \mathrm{H},=\mathrm{CH}$ and Ar), 8.02 (br s, 2H, Ar), 8.62 (br s, 1H, Ar), 9.06 (br s, 1H, Ar), 9.74 (br s, 1H, Ar), 10.34 (br s, 1H, NH), 11.07 (br s, 1H, NH), 13.18 (br s, 1H, NH). IR (KBr, cm ${ }^{-1}$ ): 3415 , 3240, 3063, 2983, 2870, 1717, 1670, 1599, 1531, 1440, 1293, 1202, 1139, 1052, 1021, 
816, 764, 692, 565. ESI-HRMS: Calcd. for $\mathrm{C}_{23} \mathrm{H}_{22} \mathrm{~N}_{6} \mathrm{O}_{4}: m / z=447,1781\left(\mathrm{MH}^{+}\right)$. Found: $m / z=$ 447,1790. Anal. Calcd. for $\mathrm{C}_{23} \mathrm{H}_{22} \mathrm{~N}_{6} \mathrm{O}_{4} \cdot \mathrm{HCl}$ : C, 57.20; H, 4.80; N, 17.40. Found: C, 56.94; H, $4.71 ; \mathrm{N}, 17.13$.

Ethyl 6-ethoxy-2-(imidazo[1,2-b]pyridazin-6-ylamino)-1-imino-8-oxo-1,2,7,8-tetrahydro 2,7-naphthyridine-4-carboxylate (13g). This compound was prepared from 4 and 6hydrazinoimidazo[1,2-b]pyridazine $\mathbf{1 0 g}$ in $\mathrm{MeOH}(2.0 \mathrm{~mL})$, reflux $2.5 \mathrm{~h}$. Yield: $120 \mathrm{mg}$ (59 \%), yellow microcrystals, m.p. $>250{ }^{\circ} \mathrm{C}$ (decomp). ${ }^{1} \mathrm{H}$ NMR (DMSO- $\left.d_{6}\right): \delta 1.30(\mathrm{t}, 3 \mathrm{H}, J=6.9 \mathrm{~Hz}$, $\left.\mathrm{OCH}_{2} \mathrm{CH}_{3}\right), 1.42$ (t, 3H, $\left.J=6.9 \mathrm{~Hz}, \mathrm{OCH}_{2} \mathrm{CH}_{3}\right), 4.35\left(\mathrm{~m}, 4 \mathrm{H}, 2 \times \mathrm{OCH}_{2} \mathrm{CH}_{3}\right), 6.97(\mathrm{~d}, 1 \mathrm{H}, J=$ $9.9 \mathrm{~Hz}, \mathrm{Ar}), 7.11(\mathrm{~s}, 1 \mathrm{H},=\mathrm{CH}), 7.58(\mathrm{~s}, 1 \mathrm{H}, \mathrm{Ar}), 7.97(\mathrm{~s}, 1 \mathrm{H},=\mathrm{CH}), 8.09$ (d, 1H, J = 9.9 Hz, Ar), 8.74 (s, 1H, Ar), 9.77 (br s, 1H, NH), 10.86 (br s, 1H, NH), 11.09 (br s, 1H, NH). IR (KBr, cm ${ }^{-1}$ ): 3417, 3210, 3073, 2985, 1720, 1670, 1597, 1553, 1482, 1397, 1292, 1216, 1188, 1049, 1017 , 815, 773, 725, 557. ESI-HRMS: Calcd. for $\mathrm{C}_{19} \mathrm{H}_{19} \mathrm{~N}_{7} \mathrm{O}_{4}: m / z=410,1577\left(\mathrm{MH}^{+}\right)$. Found: $m / z=$ 410,1591. Anal. Calcd. for $\mathrm{C}_{19} \mathrm{H}_{19} \mathrm{~N}_{7} \mathrm{O}_{4} \cdot \mathrm{HCl}$ : C, 51.18; H, 4.52; N, 21.99. Found: C, 51.00; H, $4.65 ; \mathrm{N}, 21.79$.

Ethyl 6-ethoxy-1-imino-8-oxo-2-(phthalazin-1-ylamino)-1,2,7,8-tetrahydro-2,7-naphthyridine-4-carboxylate (13h). This compound was prepared from 4 and 1-hydrazinophthalazine $(\mathbf{1 0 h})$ in $\mathrm{MeOH}(4.0 \mathrm{~mL})$, reflux $4 \mathrm{~h}$. After addition of water $(3 \mathrm{~mL})$ white precipitate was collected by filtration and washed with water. Yield: $103 \mathrm{mg}$ (49\%), white solid, m.p. 186-189 ${ }^{\circ} \mathrm{C}$ (decomp). ${ }^{1} \mathrm{H}$ NMR (DMSO-d $): \delta 1.29\left(\mathrm{~m}, 6 \mathrm{H}, 2 \times \mathrm{OCH}_{2} \mathrm{CH}_{3}\right), 4.26(\mathrm{~m}, 4 \mathrm{H}, 2 \times$ $\left.\mathrm{OCH}_{2} \mathrm{CH}_{3}\right), 6.79(\mathrm{~s}, 1 \mathrm{H},=\mathrm{CH}), 7.94(\mathrm{~m}, 3 \mathrm{H}, \mathrm{Ar}), 8.13(\mathrm{~s}, 1 \mathrm{H},=\mathrm{CH}), 8.28($ br s, $1 \mathrm{H}, \mathrm{NH}), 8.47$ $(\mathrm{m}, 2 \mathrm{H},=\mathrm{CH}$ and $\mathrm{Ar}), 11.96(\mathrm{br} \mathrm{s}, 1 \mathrm{H}, \mathrm{NH}), 12.59(\mathrm{~s}, 1 \mathrm{H}, \mathrm{NH}) .{ }^{13} \mathrm{C} \mathrm{NMR}\left(\mathrm{DMSO}-d_{6}\right): \delta 13.9$, $61.6,66.4,83.0,101.2,110.2,116.0,126.0,129.2,143.7,146.1,147.9,155.8,160.9,162.3$, 162.7. IR (KBr, cm $\left.{ }^{-1}\right): 3410,2982,1715,1645,1593,1545,1479,1445,1382,1272,1178,1148$, 1032, 926, 814, 762, 670, 597. ESI-HRMS: Calcd. for $\mathrm{C}_{21} \mathrm{H}_{20} \mathrm{~N}_{6} \mathrm{O}_{4}: m / z=421.1624\left(\mathrm{MH}^{+}\right)$. Found: $m / z=421.1618$. Anal. Calcd. for $\mathrm{C}_{21} \mathrm{H}_{20} \mathrm{~N}_{6} \mathrm{O}_{4}$ : C, 59.99; H, 4.79; N, 19.99. Found: C, 59.71; H, 4.77; N, 19.71 .

Ethyl 6-ethoxy-1-imino-8-oxo-2-(pyridine-2-ylamino)-1,2,7,8-tetrahydro-2,7-naphthyridine4-carboxylate (13i). This compound was prepared from 4 and 2-hydrazinopyridine (10i) in EtOH (1.5 mL), reflux 1 h. Yield: $59 \mathrm{mg}$ (32\%), white solid, m.p. 246-248 ${ }^{\circ} \mathrm{C}$ (decomp). ${ }^{1} \mathrm{H}$ NMR (DMSO-d $): \delta 1.26\left(\mathrm{~m}, 6 \mathrm{H}, 2 \times \mathrm{OCH}_{2} \mathrm{CH}_{3}\right), 4.24\left(\mathrm{~m}, 4 \mathrm{H}, 2 \times \mathrm{OCH}_{2} \mathrm{CH}_{3}\right), 6.68(\mathrm{~s}, 1 \mathrm{H}$, $=\mathrm{CH}), 6.84(\mathrm{~d}, 1 \mathrm{H}, J=8.4 \mathrm{~Hz}, \mathrm{Ar}), 6.97\left(\mathrm{dd}, 1 \mathrm{H}, J_{1}=6.9 \mathrm{~Hz}, J_{2}=5.1 \mathrm{~Hz}, \mathrm{Ar}\right), 7.73\left(\mathrm{ddd}, 1 \mathrm{H}, J_{1}\right.$ $\left.=8.1 \mathrm{~Hz}, J_{2}=6.6 \mathrm{~Hz}, J_{3}=1.8 \mathrm{~Hz}, \mathrm{Ar}\right), 7.92(\mathrm{~s}, 1 \mathrm{H},=\mathrm{CH}), 8.14$ (br dd, $\left.1 \mathrm{H}, J=5.1 \mathrm{~Hz}, \mathrm{Ar}\right), 8.68$ (br s, 1H, NH), 10.04 (br s, 1H, NH), 12.41 (s, 1H, NH). IR (KBr, cm ${ }^{-1}$ ): 2980, 1720, 1707, 1649, 1603, 1545, 1477, 1441, 1423, 1381, 1292, 1279, 1246, 1176, 1144, 1098, 1032, 927, 831, 774. EI-HRMS: Calcd. for $\mathrm{C}_{18} \mathrm{H}_{19} \mathrm{~N}_{5} \mathrm{O}_{4}: m / z=369.1437\left(\mathrm{M}^{+}\right)$. Found: $m / z=369,1443$. Anal. Calcd. for $\mathrm{C}_{18} \mathrm{H}_{19} \mathrm{~N}_{5} \mathrm{O}_{4}$ : C, 58.53; H, 5.18; N, 18.96. Found: C, 58.23; H, 5.01; N, 18,71 .

\section{X-Ray structure analysis for compound (6b)}

Single crystal X-ray diffraction data of compound $\mathbf{6 b}$ were collected at room temperature on a Nonius Kappa CCD diffractometer using the Nonius Collect Software. ${ }^{11}$ DENZO and 
SCALEPACK ${ }^{12}$ were used for indexing and scaling of the data and the structures were solved by means of SIR97. ${ }^{13}$ Refinement was done using Xtal3.6 ${ }^{14}$ program package and the crystallographic drawing was prepared by ORTEP-3. ${ }^{15}$ Crystal structure was refined on $F$ values using the full-matrix least-squares procedure. The non-hydrogen atoms were refined anisotropically, while the positions of hydrogen atoms were geometrically calculated and their positional and isotropic atomic displacement parameters were not refined. Crystallographic data (excluding structure factors) have been deposited with the Cambridge Crystallographic Data Centre as supplementary material with the deposition number CCDC 796256. Copies of the data can be obtained, free of charge via http://www.ccdc.cam.ac.uk/conts/retrieving.html.

\section{Acknowledgements}

The financial support from the Slovenian Research Agency, Slovenia through grants P0-05020103, P1-0179, and J1-6689-0103-04 is gratefully acknowledged. Financial support by the pharmaceutical companies $L E K-S A N D O Z$, Ljubljana, and $K R K A$, Novo mesto, is fully appreciated.

\section{References}

1. Šimunek, P.; Baškovč, J.; Grošelj, U.; Meden, A.; Svete, J.; Stanovnik, B. Z. Naturforsch. 2010, 65b, 807 .

2. For reviews see: (a) Stanovnik, B. J. Heterocycl Chem. 1999, 36, 1581. (b) Stanovnik, B.; Svete, J. Targets in Heterocycl. Syst. 2000, 4, 105. (c) Stanovnik, B.; Svete, J. Synlett 2000, 1077. (d) Stanovnik, B.; Svete, J. Chem. Revs. 2004, 104, 2433. (e) Stanovnik, B.; Svete, J. Mini-Reviews in Organic Chemistry 2005, 2, 211. (f) Svete, J.; Monatsh. Chem. 2004, 135, 629. (g) Bevk, D.; Svete, J.; Stanovnik, B. In Modern Approaches to the Synthesis of O- and N-Heterocyles, Vol. 3, Kaufmann, T. S.; Larghi, E. L., Eds., Research Signpost, Trivandrum (India), 2007, Chapter 3, pp 73.

3. For recent publications see: (a) Stanovnik, B.; Smodiš, J. Tetrahedron 1998, 54, 9799. (b) Pirc, S.; Bevk, D.; Jakše, R.; Rečnik, S.; Golič, L.; Golobič, A.; Meden, A.; Stanovnik, B.; Svete, J. Synthesis 2005, 2969. (c) Pirc, S.; Bevk, D.; Golobič, A.; Stanovnik, B.; Svete, J. Helv. Chim. Acta 2006, 89, 30. (d) Uršič, U.; Grošelj, U.; Meden, A.; Svete, J.; Stanovnik, B. Tetrahedron Lett. 2008, 49, 3775. (e) Uršič, U.; Svete, J.; Stanovnik, B. Tetrahedron 2008, 64, 9937. (f) Uršič, U.; Grošelj, U.; Meden, A.; Svete, J.; Stanovnik, B. Helv. Chim. Acta 2009, 92, 481. (g) Uršič, U.; Grošelj, U.; Meden, A.; Svete, J.; Stanovnik, Synthesis 2009, 217.

4. (a) Strah, S.; Stanovnik, B.; Golič Grdadolnik, S. J. Heterocycl. Chem. 1997, 34, 263. (b) Selič, L.; Stanovnik, B. Helv. Chim. Acta 1998, 81, 1634. (c) Selič, S.; Jakše, R.; Lampič, 
K.; Golič, L.; Golič Grdadolnik, S.; Stanovnik, B. Helv. Chim Acta 2000, 83, 2802. (d) Jakše, R.; Rečnik, S.; Svete, J.; Golobič, A.; Golič, L.; Stanovnik, B. Tetrahedron 2001, 57 , 8395. (e) Grošelj, U.; Rečnik, S.; Svete, J.; Meden, A.; Stanovnik, B. Tetrahedron: Asymmetry 2002, 13, 821. (f) Čebašek, P.; Wagger, J.; Bevk, D.; Jakše, R.; Svete, J.; Stanovnik, B. J. Comb. Chem. 2004, 6, 356. (g) Jakše, R.; Bevk, D.; Golobič, A.; Svete, J.; Stanovnik, B. Z. Naturforsch. 2006, 61b, 413. (h) Časar, Z.; Bevk, D.; Svete, J.; Stanovnik, B. Tetrahedron 2005, 61, 7508. (i) Wagger, J.; Bevk, D.; Meden, A.; Svete, J.; Stanovnik, B. Helv. Chim. Acta 2006, 89, 240. (j) Jakše, R.; Grošelj, U.; Soršak, G.; Svete, J.; Stanovnik, B. Heterocycles 2007, 73, 743. (k) Jakše, R.; Golič, L.; Meden, A.; Grošelj, U.; Svete, J.; Stanovnik, B. Heterocycles 2007, 74, 293. (1) Wagger, J.; Grošelj, U.; Meden, A.; Svete, J.; Stanovnik, B. Tetrahedron 2008, 64, 2801. (m) Malavašič, Č.; Wagger, J.; Stanovnik, B.; Svete, J. Tetrahedron: Asymmetry 2008, 19, 1557. (n) Wagger, J.; Svete, J.; Stanovnik, B. Synthesis 2008, 143.

5. Ikekawa, N. Chem. Pharm. Bull. 1958, 6, 263.

6. (a) Lowe, P. A. In Comprehensive Heterocyclic Chemistry: Vol. 2, 581. (b) Stanforth, S. P. In Comprehensive Heterocyclic Chemistry Vol 7, Pergamon 1996, 527. (c) Dobbs, A. P. in Comprehensive Heterocyclic Chemistry, Vol 10, Amsterdam 2008, 713. (d) Phuan, P.-W.; Kozlowski, M. C. In Science of Synthesis, Vol 15, Georg Thieme Verlag, Stuttgart, New York 2006, 947. (e) Litvinov V. P. Adv. Heterocycl. Chem. 2006, 91, 11189.

7. Baldwin, J. J.; Mensler, K.; Ponticello, G. S. J. Org. Chem. 1978, 43, 4878.

8. Tan, R.; Taurins, A. Tetrahedron Lett. 1966, 7, 1233.

9. Numata, A.; Kondo, Y.; Sakamoto, T. Synthesis 1999, 306.

10. van den Haak, H. J. W.; van der Plas, H. C.; Veldhuizen, B. J. Heterocycl. Chem. 1981, 18, 1349.

11. Collect Software. Nonius, BV, Delft, The Netherlands, 1998.

12. Otwinowski, Z.; Minor, W. Methods Enzymol. 1997, 276, 307.

13. Altomare, A.; Burla, M. C.; Camalli, M.; Cascarano, G. L.; Giacovazzo, C.; Guagliardi, A.; Moliterni, A. G. G.; Polidori, G.; Spagna, R. J. Appl. Crystallogr. 1999, 32, 115.

14. Hall, S. R.; du Boulay, D. J.; Olthof-Hazekamp, R. Eds. Xtal3.6 System. 1999.

15. Farrugia, L. J. J. Appl. Cryst. 1997, 30, 565. 